\title{
Modelo teórico para la integración de las emociones en el campo del comportamiento organizacional
}

\author{
Theoretical model for the integration \\ of emotions in the field \\ of organizational behavior
}

\author{
Gonzalo Flores-Castro Lingán \\ Universidad Católica San Pablo, Arequipa, Perú \\ gaflores@ucsp.edu.pe
}

\section{Resumen}

Las emociones tienen un papel muy importante en las organizaciones empresariales, desde la influencia en la actitud y conducta de sus miembros hasta la medición del éxito de la misma. Lamentablemente, las dificultades para el estudio de las emociones son varias; destaca la dificultad de integración de las emociones en el campo del comportamiento organizacional. El presente artículo intenta abordar dicho problema desde el punto de vista de la toma de decisiones, utilizando el modelo teórico propuesto por Juan Antonio Pérez López, el cual se encuadra dentro del paradigma del management cybernetics. El aporte del trabajo está dado en mostrar cómo el modelo de Pérez López logra introducir la variable emoción o afecto desde el interior de la teoría, a través de diferentes constructos (motivación espontánea, satisfacción percepcional, satisfacción estructural, etc.) que permiten una explicación mínima pero valiosa del comportamiento organizacional.

\section{Palabras clave}

Comportamiento organizacional - integración de emociones - toma de decisiones

\section{Abstract}

Emotions have a very important role in business organizations, from the influence on the attitude and behavior of its members to the measurement of its success. Unfortunately, the difficulties for the study of emotions are several, especially the difficulty of integrating 
emotions in the field of organizational behavior. This article attempts to address that problem from the point of view of decision-making using the theoretical model proposed by Juan Antonio Pérez López, which is framed in the management cybernetics paradigm. The contribution of this work is to show how Pérez Lópezıs model manages to introduce the variable emotion or affection from within the theory, through different constructs (spontaneous motivation, perceptional satisfaction, structural satisfaction, etc.) that allow a minimal but valuable explanation of organizational behavior.

\section{Keywords}

Organizational behavior - integration of emotions - decision making

\section{Introducción}

En la actualidad se reconoce el papel preponderante que tienen las emociones en las organizaciones empresariales (Kangasharju y Nikko, 2009). Entre las diversas razones se encuentra su influencia en la actitud y conducta de los miembros de la organización (Lee y Allen, 2002), una mayor precisión en la toma de decisiones (Staw y Barsade, 1993), una menor probabilidad de rotación (Locke, 2009a) o la medición del éxito de una organización (Caruso, 2001). Sin embargo, a pesar de que su estudio en las organizaciones no es algo nuevo (Mastenbroek, 200o), y que el interés por la investigación de las emociones en la empresa haya incrementado considerablemente, la investigación empírica ha sido de lenta aplicación (Ashkanasy, 2015) debido a diversas dificultades en el objeto de estudio (Locke, 2009a).

Las dificultades son variadas, por lo que, sin ánimo de ser exhaustivo, podemos mencionar las siguientes: la centralidad del homo economicus que aún domina la economía y la dirección de empresas, cuyo «agente racional» es movido por el interés personal, no considerando las emociones salvo de manera negativa, omitiendo la relación entre emociones y racionalidad (Melé y González Cantón, 2015); la falta de definición de lo que es la emoción, debido a la naturaleza introspectiva de la misma, cuyo estudio ha sido erróneamente proscrito — según Locke (2009a) - del campo de la psicología por más de cien años dada la hegemonía del conductismo entre otras razones (Locke, 2009b); o la dificultad de integración de las emociones en el campo del comportamiento organizacional (Grandey, 20o8).

Esta última dificultad - la integración de las emociones - es la que intentaremos abordar teóricamente en el presente trabajo, en especial en lo que respecta a la toma de decisiones, puesto que son estas las que determinan el comportamiento de las personas (Pérez López, 1991). El tema es complejo, pues aborda problemas epistemológicos en toda la ciencia del management (Rosanas, 2007). Sin entrar en la discusión filosófica, pasaremos primero a la exposición del problema de integración y luego a la elaboración del modelo, el cual iremos construyendo de manera que se observe cómo la variable emoción o afecto es integrada desde el interior de la teoría, no siendo un mero agregado accidental, sino un constructo necesario 
para la explicación del comportamiento organizacional.

\section{El problema de integración}

La integración de las emociones representa un desafío para el comportamiento organizacional puesto que su estudio no pasa por aspectos que sean observables directamente, ni siquiera por el propio agente que siente. De hecho, se suele decir que es irreducible a ser objetivado intelectualmente, como comenta Fabro (1982, p. 110), «la naturaleza íntima del sentimiento no es objeto de definiciones, sino de la experiencia, porque el sentimiento es un prius absoluto que escapa a todo análisis reflexivo y solo se deja describir de modo indirecto». Para enfrentar este problema en el comportamiento humano se introducen nociones como la de «inteligencia emocional», pero resultan problemáticas conceptualmente, ya que se estaría hablando del uso de la inteligencia sobre las emociones y no de un tipo de inteligencia aparte (Locke, 2005). En realidad, afirma Brennan (1969, p. 247), «el intelecto y la sensibilidad se hallan relacionados, pero uno no puede ser explicado en términos del otro».

Por otra parte, se intenta clarificar el asunto tratando de proporcionar definiciones como las siguientes: «las emociones son la forma en que una persona experimenta juicios devalor de manera automática o subconsciente» (Locke, 2009a, p. 145) o «valoración de una realidad externa respecto de los propios deseos o impulsos (instintivos o tendenciales)» (Choza, 1988, p. 222). Aunque esto ayudea la comprensión de su realidad, carece de la operativización suficiente para la ciencia del management (en la que se inserta el campo del comportamiento organizacional), a la cual compete aquellas verdades útiles que le permitan resolver sus problemas específicos (Rosanas, 2006)ํ․ Además, se procuran modelos que, si bien son útiles, tratan de manera aislada la emoción, sin tomar en cuenta cómo se inserta esta en el dinamismo del comportamiento organizacional. Por ejemplo, el patrón universal de toda emoción (exceptuando aquellos causados por estados cerebrales anómalos o desbalances hormonales) es: objeto $\rightarrow$ cognición $\rightarrow$ estimación del valor $\rightarrow$ emoción. El objeto puede ser una persona, una acción, un evento, una idea, un recuerdo o una emoción previa. La cognición involucra la percepción del objeto, el contexto además de todo conocimiento previo que el subconsciente automáticamente asocia con el objeto. La valoración involucra una «medición» subconsciente del objeto con respecto a lo que valoramos internamente (Cfr. Locke, 2009a, p. 146). Esto puede resultar de una utilidad enorme si nos enfocamos solamente en la emoción y cómo esta puede encausarse, como es el caso de la terapia cognitivo-conductual (Locke, 2009a), pero deja a criterio del profesional o del investigador el cómo se relaciona la emoción con, por ejemplo, la toma de decisiones, el uso del poder, el liderazgo, etc.

Es aquí donde entra la propuesta teórica de Juan Antonio Pérez López, cuyo modelo teórico, relativamente poco conocido, viene siendo aplicado en diversas áreas

\footnotetext{
1 Nótese que esto no es un utilitarismo epistemológico. No afirmamos que el criterio de verdad sea la utilidad, es decir, «es verdadero porque es útil»; afirmamos algo distinto, «de lo verdadero, lo útil». Es decir, se usan abstracciones de realidades con ciertos fines utilitarios, pero siguen partiendo de la realidad y se ajusta a ella, tomando todas las variables relevantes al problema.
} 
del management como la toma de decisiones (Ariño, 2005; Rosanas, 2007; Lopez Jurado, 2010), el liderazgo (Âlvarez de Mon, Cardona, Chinchilla, Pin et al., 2001; Gorrochotegui, 2007), el uso del poder (Pérez López, 1991; Ferreiro y Alcázar, 2012), la ética empresarial (Argandoña, 2008; Flores-Castro, 2018), la motivación (García A. T., 2004), la selección de personal y la comunicación organizacional (Ferreiro y Alcázar, 2012). El modelo de Pérez López es una propuesta teórica que se encuadra en el paradigma del management cybernetics (Melé, Chinchilla, y López-Jurado, 2018) y estudia la acción humana de tal manera que permite la integración, desde el interior de la teoría, de las diversas materias propias del campo del comportamiento organizacional. Poco se ha escrito sobre uno de sus aportes más interesantes, la integración de la emoción en la acción humana dentro de las organizaciones, por lo que este trabajo representa un aporte en ese ámbito. Pasaremos a la exposición de la propuesta perezlopiana que tomará como base el mínimo requerido para toda organización: la interacción de dos agentes personales. Para ello, se construirá el modelo utilizando aportes de otras ciencias que permitan explicar cómo se introducen los afectos en la acción personal y organizacional.

\section{Evaluación de la acción humana}

El esquema mínimo para conceptualizar la interacción (acción-reacción) entre dos personas puede graficarse según se muestra en la figura 1.

Cuando el agente activo tiene un problema, es decir, se percata que «existe una situación que no es del todo agradable para una persona [él mismo u otra persona], o cuando se sospecha que existen situaciones más agradables que las actuales» (Ariño, 2005, p. 19), y que no puede solucionar solo; ejecuta una acción con otro agente (reactivo), con la esperanza de que la acción de dicho agente (la reacción) solucione su problema.

Si realizamos un rápido análisis de esta interacción caemos en cuenta que tenemos, al menos, tres evaluaciones que debemos realizar para tratar de conocer a priori la eficacia de la acción del agente activo. En primer lugar, se debe analizar la proposición, o plan de acción, la díada «acción-reacción»; esto es, evaluar la validez de la proposición, que no es otra cosa que

Figura 1

Modelo básico de sistema

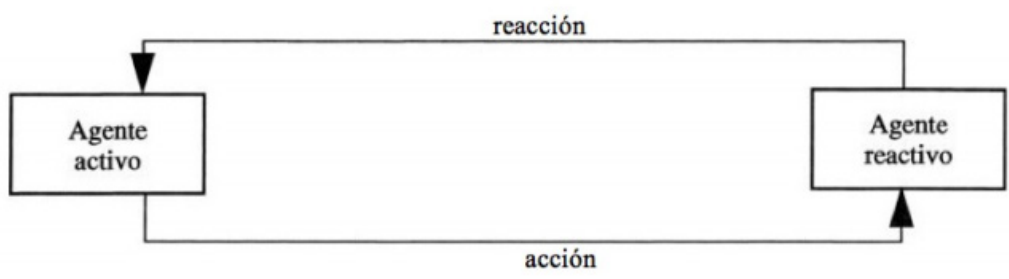

Nota: Original de Teoría de la acción en las organizaciones: la acción personal de Pérez López, J. A., 1991, p. 25. 
«la medida en que la acción y la reacción realmente resuelven el problema que se pretendía resolver» (Ariño, 2005, p. 30). En segundo lugar, debemos evaluar si el estado interno del agente activo es tal que le permite ejecutar el plan de acción; es decir, si le es factible u operativo ejecutar el plan de acción. Por último, hemos de evaluar si el estado interno del agente reactivo está en condiciones de realizar la reacción; o sea, si le es operativo o instrumental (operatividad del agente reactivo) para dicho agente ejecutar su acción (la reacción).

Esta evaluación de una acción concreta es lo que podemos llamar una evaluación estática; sin embargo, en las organizaciones se requieren múltiples interacciones entre diversos agentes en distintos momentos, sea entre directivos y colaboradores, colaboradores con otros colaboradores, vendedores con clientes, etc. ¿Qué pasaría si los estados internos de los agentes cambian debido a las interacciones pasadas? La pregunta es clave para realizar un análisis completo. De hecho, la experiencia muestra que ese cambio existe y que influencia en las futuras interacciones, incluso a nivel neuronal (Sapolsky, 2017). No tomar en cuenta el cambio de los estados internos de los agentes para la evaluación de una acción sería una abstracción incompleta. En ese sentido, al cambio producido en el agente activo luego de una interacción le llamaremos eficiencia - operatividad futura-, y al cambio producido en el agente reactivo le llamaremos consistencia - instrumentalidad futura-.

Ahora bien, el cambio al que nos referimos no es cualquier tipo de cambio, sino aquel «cambio que ocurra en los agentes como consecuencia de la ejecución del proceso de interacción, siempre que dicho cambio sea significativo para la explicación de las futuras interacciones» (Pérez López, 1991). Para distinguirlo utilizaremos el término aprendizaje, que es mucho más preciso. Dicho aprendizaje puede ser positivo, es decir, permite que las interacciones puedan continuar produciéndose en el futuro (o incluso facilitar las interacciones futuras); o negativo, esto es, un aprendizaje que dificulte o incluso imposibilite las futuras interacciones. Así, tenemos la evaluación mínima completa de una interacción: un análisis estático (eficacia del plan de acción) y un análisis dinámico, eficiencia y consistencia de un plan de acción.

\section{Resultados y motivos de la acción}

Teniendo en cuenta la figura 1 y el análisis realizado, podemos afirmar que por cada decisión, o plan de acción, se pueden esperar tres tipos de consecuencias de la acción o resultados de la acción (Pérez López, 2014): resultados extrínsecos, o la misma acción del agente activo y la reacción del agente reactivo (lo que ambos hacen, la propia interacción) ${ }^{2}$; resultados intrínsecos, el aprendizaje del agente activo fruto de la decisión tomada; y resultados trascendentes, o el aprendizaje del agente reactivo fruto de la interacción. Estos resultados son «irreducibles entre sí» (Alcázar, 2010, p. 105), además de ser $a$ posteriori, mientras que la evaluación del plan de acción por parte del agente activo es a priori.

La consideración de los resultados de la acción nos lleva a la valoración de estos para la

2 Nótese que la operacionalidad e instrumentalidad actuales no son resultado de la interacción, sino sus condiciones de posibilidad. 
decisión. En otras palabras, «el logro de cualquiera de aquellos tres tipos de resultados, o de todos simultáneamente, puede llegar a ser motivo de las decisiones de una persona, es decir, puede ser un logro intentado en sus decisiones»3 (Pérez López, 2014, p. 55). Este sencillo esquema lógico nos muestra que los agentes pueden tener distintos motivos para la acción. Al respecto, Pérez López (2014, p. 55) afirma que «[los] aprendizajes pueden tener un gran valor para el agente activo... [No siendo] nada extraño que la consecución de dichos aprendizajes pueda ser un objetivo explícitamente buscado por las decisiones de una persona», pero añade inmediatamente que «también puede ocurrir que ambos aprendizajes no solo no sean buscados, sino que sean, por el contrario, ignorados a la hora de tomar las decisiones» (Pérez López, 2014, p. 55). De hecho, nada nos impide pensar que, en toda lógica, el agente activo tenga que actuar por todos los motivos al mismo tiempo; sin embargo, «en una acción intervienen ordinariamente los tres motivos, aunque con mayor intensidad unos que otros» (Ferreiro y Alcázar, 2012, p. 65).

Tenemos por tanto tres motivos de la acción para agente: motivos extrínsecos o aquellos que buscan los resultados extrínsecos, es decir, el logro de satisfacciones - la resolución del problema que producen las interacciones-; motivos intrínsecos o la valoración de los resultados intrínsecos, a saber, la búsqueda del propio aprendizaje del agente activo; y los motivos trascendentes o aquellos que buscan resultados trascendentes, o sea, el

3 Motivo es la realidad en cuanto se prevé que resolverá el problema del agente, siendo por tanto valorada. En ese sentido, motivo y valor son lo mismo (Ferreiro y Alcázar, 2012). aprendizaje del agente reactivo ${ }^{4}$. Cabe notar que, al realizar una acción, el agente activo puede tener un motivo ignorando otros, pero los resultados se dan con independencia de las intenciones del decisor.

\section{Relación estructural, relación opera- tiva y tipos de aprendizaje}

Asimismo, dado el análisis anterior, podemos deducir que se encuentran dos tipos de relaciones entre los agentes, una subyacente y necesaria para la otra. Por una parte, podríamos hablar de las condiciones de posibilidad de la interacción (acción-reacción): el estado interno de los agentes - operacionalidad e instrumentalidad-debe ser tal (debe haber un mínimo necesario) que permita la interacción; de no ser así, no hay interacción posible. A esta relación subyacente le llamaremos relación estructural, esto es, «los grados de confianza que puedan existir entre ambas personas» (Pérez López, 2014, p. 156). Supuesta esta relación estructural (entre otras cosas, un mínimo de confianza), la relación toma otro cariz, el de la relación operativa o aquellas acciones y reacciones que de hecho solucionan los problemas del agente activo y del reactivo. Nótese que tanto la relación estructural como la relación operativa son solo abstracciones de la relación real u organización real. Si la organización, la relación real, desea

4 La búsqueda del aprendizaje del agente activo (resultado intrínseco) es un motivo intrínsecoy produce el resultado intrínseco, mientras que la búsqueda del resultado trascendente es un motivo trascendente que produce un resultado intrínseco no buscado directamente (un aprendizaje en el agente activo que le facilita la consideración de resultados trascendentes), no produce el resultado trascendente (esto depende también del agente reactivo). 
seguir en el tiempo, se ha de tomar decisiones tomando en cuenta los criterios de eficiencia y consistencia, es decir, velando por que los estados internos de los agentes tomen en cuenta los resultados intrínsecos y trascendentes de las acciones (como condición de posibilidad de cualquier interacción actual y futura), y que mejoren en la calidad de sus interacciones, a saber, que las acciones y reacciones solucionen cada vez mejor los problemas.

Asimismo, dado que los motivos de la acción son lógicamente distintos, pueden existir al menos dos tipos de aprendizaje. Si me mueven a actuar los motivos extrínsecos estoy buscando mejorar la relación operativa (¿qué tipo de acción generará la reacción que busco?, ¿cómo puedo mejorar la eficacia de mis acciones para generar mejores reacciones?), tendré un resultado intrínseco (buscado o no) que es un aprendizaje operativo, «adquisición de un conocimiento o habilidad "técnica”: aprender un idioma, manejar un auto, dominar una técnica financiera... etc.» (Ferreiro y Alcázar, 2012, p. 61). Por otra parte, si me mueve a actuar motivos trascendentes estoy buscando la mejora en la relación estructural (¿el otro querrá seguir colaborando conmigo a futuro?, ¿lo que estoy haciendo está fortaleciendo nuestra relación?, ¿le estoy tomando en cuenta?, ¿le ayudo a tomarme en cuenta en su decisión?), tendré un resultado intrínseco (querido o no) que es un aprendizaje estructural, se aprende «a tomar conciencia del impacto de [la] acción sobre otras personas» (Ferreiro y Alcázar, 2012, p. 62). Debe tomarse en cuenta que tanto «(a)prendizaje operativo y estructural son los dos tipos de resultados internos que se producen en la persona por el solo hecho de haber actuado, con independencia de lo que venga del exterior o de lo que les ocurra a las personas con las que interactúa»5 (Ferreiro y Alcázar, 2012, p. 62); además, ambos pueden ser tanto positivos como negativos.

\section{El proceso de toma de decisiones}

Ahora bien, ¿cómo debe estar hecho por dentro el agente activo (la persona) para poder evaluar todas estas variables (eficacia, eficiencia y consistencia)? En este punto trataremos brevemente de explicar los mecanismos necesarios

5 ¿Cabe actuar buscando los resultados intrínsecos (motivos intrínsecos)? Sí, pero el asunto es problemático. Como se verá, en el proceso de toma de decisiones no puede existir un impulso espontáneo hacia algo concreto, pero sí un impulso genérico a aprender que Pérez López llama motivación potencial. La diferencia puede verse claramente en la distinción que hace la psicología clásica entre apetito natural y apetito elícito: dada la situación orgánica de una persona, esta sentirá hambre - apetito natural-, pero no hambre de algo concreto, simplemente hambre genérica (problema); buscará comer algo y, de tener éxito, lo estimará positivo y conservará en su memoria (memoria percepcional), buscando repetir automáticamente la interacción (apetito elícito sensible) cuando el problema vuelva a aparecer. El caso del ser humano es distinto, posee inteligencia, la cual tiene un apetito natural hacia el aprendizaje abstracto genérico que le lleva a mejorar como persona (desea la verdad y por tanto el bien, la motivación potencial); luego de interactuar, de tener éxito, puede buscar actuar por su conocimiento experimental (que modifica la motivación espontánea), siendo algo que ya está en la memoria. Cualquier aprendizaje futuro solo es genérico (no hay memoria de lo que no se conoce), aunque puede tomar como ejemplo lo ya conocido por su conocimiento experimental ( $\mathrm{Cfr}$. Flores-Castro, 2018). 
- los constructs - para una evaluación completa de la decisión personal.

Al actuar el agenteactivo (o el reactivo) buscan resolver un problema, es decir, lograr una satisfacción. Para ilustrar, consideremos a un agente más simple, un antílope africano o ñu, este agente puede ir perdiendo energía en el transcurso del día ${ }^{6}$, por tanto, verse motivado a buscar comida (siente hambre). Esa fuerza o impulso automático, dada la programación inicial del agente (su código genético, las leyes de la física, etc.) le llamaremos motivación espontánea. El ñu entrará en acción buscando interactuar con su entorno (el agente reactivo) para solucionar su problema. Encontrará quizás un nuevo pastizal y procederá a comer; al hacerlo resolverá su problema, logrará una satisfacción que detendrá la motivación y, por tanto, la acción. Dicha satisfacción parte de un conocimiento percepcional obtenido como consecuencia de la interacción, o sea, es provocado por la observación externa. Este tipo de satisfacción percepcional se corresponde con los resultados extrínsecos de la acción, los cuales provocan un aprendizaje -memoria percepcional- que modifica la motivación espontánea futura. En el caso del ñu, podría ser que el tipo de pasto que comió tenía algún tipo de veneno que le causó una insatisfacción, lo cual queda guardado en su memoria percepcional y que modifica sus acciones futuras ante distintos pastizales (sufre un cambio en sus preferencias percepcionales $\left.{ }^{7}\right)$.

6 Sin siquiera hacer nada, simplemente por la tendencia hacia la entropía de cualquier sistema físico (Carroll, 2017).

7 Estas preferencias están dadas por su programación inicial (genética) y sus aprendizajes. Debido a distintas mutaciones genéticas o diferencias en los aprendizajes los agentes actúan como actúan
En nuestro ejemplo del ñu, los mecanismos son suficientes para la solución de sus problemas con independencia de la solución de sus problemas futuros. En otras palabras, el ñu no evalúa los resultados intrínsecos y trascendentes de sus acciones. Podría suceder que, dada una situación, elija actuar de una manera que destruya su entorno (acción inconsistente extrema) para satisfacer sus problemas actuales sin importarle los futuros - como en el caso de depredar todo el pastizal de una zona - y tenga que buscar otro entorno (agente reactivo) con el que interactuar o que simplemente perezca. Por todo lo que sabemos, al ñu no parece importarle mucho que esto suceda, como tampoco al electrón al interactuar con un protón, etc. Sin embargo, al ser humano sí parece importarle el asunto. El agente activo personal intenta cuidar al agente reactivo para poder seguir interactuando y resolver sus problemas ahora y en el futuro, es decir, evalúa los resultados intrínsecos y trascendentes de sus acciones.

Para evaluar los resultados intrínsecos y trascendentes se requieren otros mecanismos distintos. Esto sucede porque, como dice Ariño (2005, p. 31) «[tanto] la validez como la operacionalidad de la decisión son directamente observables para el agente activo; sin embargo, la instrumentalidad no lo es, puesto que está en el interior del agente reactivo»; en otras palabras, no se puede percibir directamenteel estado internoactual del agente reactivo (instrumentalidad), su estado interno potencial (consistencia), ni

(o deciden como deciden). Lo mismo pasa con la persona humana; sin embargo, pensamos que en el ser humano esas preferencias percepcionales caracterizan a cada persona como individuo y le son propias e incomunicables (Ariño, 2005) - una manifestación propia del ser persona, la intimidad (García J. Á., 2014)—. 
la operacionalidad futura (eficiencia) del agente activo, esto último debido a que «los resultados intrínsecos ocurrirán en el interior del agente activo y aún no han tenido lugar, por lo que no se pueden recordar» (Ariño, 2005, p. 51). Este hecho puede ser graficado con el siguiente ejemplo: un amigo nuestro acaba de cerrar la puerta de su auto y se ha golpeado la mano al hacerlo, nosotros vemos que grita pero no percibimos su dolor ${ }^{8}$; a la inversa, si fuésemos nosotros los que nos hubiésemos golpeado la mano, percibiríamos el dolor, pero nuestro amigo solo podría escuchar nuestro grito (Feser, 2006).

El agente activo requiere, antes que nada, darse cuenta de que la alternativa proporcionada por la motivación espontánea es insuficiente para solucionar sus problemas; esto debido a que procede de una evaluación

8 Podría ser, en algún caso, que percibiéramos placer por lo que acabamos de ver. incompleta (solo toma en cuenta resultados extrínsecos). A este darse cuenta le llamaremos motivación racional pues procede de un conocimiento racional de lo que se está realizando. Esta motivación no es una fuerza o impulso, no es operativa o tendida a la acción como la motivación espontánea, es simplemente una toma de consciencia. Para hacerla operativa requerimos de dos variables de control, la racionalidad y la virtualidad, que explicaremos mediante la figura 2.

El agente activo realiza una determinada acción dado su conocimiento percepcional/memoria percepcional (preferencias percepcionales) e impulsado por su motivación espontánea. En este caso, solo evalúa los resultados extrínsecos de su acción (la eficacia de su acción). Sin embargo, se da cuenta de que la acción podría tener efectos futuros para sí mismo o para el agente reactivo (conocimiento racional/motivación racional). Para operativizar la motivación

Figura 2

\section{Motivación racional y espontánea}

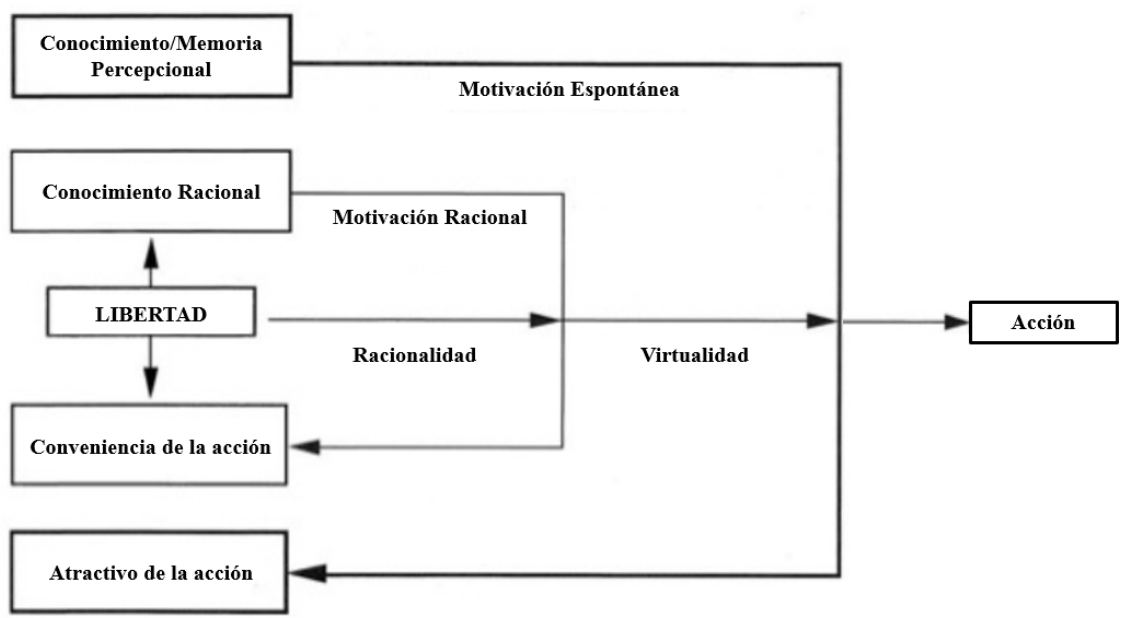

Nota: Modificado del original de Fundamentos de la dirección de empresas de Pérez López, J. A., 2014, p. 152. 
racional se requiere de una fuerza, que en este caso es la libertad humana9, «ejerciendo el oportuno control sobre el impulso de la motivación espontánea» (Pérez López, 2014, p. 151), para luego generar planes de acción alternativos, distintos al que propone la motivación espontánea, y que consideren los resultados intrínsecos (eficiencia) y trascendentes (consistencia) de la decisión —racionalidad_, y que controle el impulso de la motivación espontánea e imponga la alternativa conveniente — virtualidad-.

\section{Los afectos o emociones en la acción humana}

Hasta este punto, los mecanismos de los agentes son suficientes para poder establecer de manera lógica un sistema de interacciones coherente y estable en el tiempo. Sin embargo, dicha estabilidad parece requerir de un esfuerzo constante por parte de los agentes puesto que la motivación racional requiere estar controlando el impulso de la

9 Según Pérez López (2014, p. 152) la «libertad es la capacidad de las personas para generar una energía que les permite autodeterminarse a la hora de actuar». La libertad es un supuesto de todo el sistema de Pérez López (Alcázar, 2010), el cual cuenta con sólidos fundamentos filosóficos (Mele, 2011, 2014) y científicos (Fuster , 2016). Según Melé, Chinchilla y López-Jurado (2018), «Pérez López did not debate the so-called problem of free will (is free will real or a mere illusion?), but his implicit assumption-like Aristotle-is that free will is not an illusion and we are not fully determined by external or biological factors». El tema es discutible, pero afirmamos junto con Fuster (2016), que «es precisamente en el crisol de probabilidades e incertidumbres del cerebro humano donde cobra vida la libertad. La capacidad para escoger entre posibilidades proviene literalmente de la varianza y los grados de libertad de innumerables variables que subyacen a la acción humana futura. Como pasa con la evolución, el determinismo y la causalidad directa se disuelven en la probabilidad y, al hacerlo, ceden ante un factor teleológico: la finalidad, el objetivo» (p. 17). motivación espontánea e imponer las alternativas más convenientes (podría decirse que es pura fuerza de voluntad). No obstante, la experiencia humana muestra que esto no tiene por qué ser así. La vida afectiva del ser humano es precisamente esa variable que ayuda al ser humano en su actuar, pudiendo encontrarse incluso emociones que son solo humanas ${ }^{10}$ (Cfr. Scheler, 1942; Rodríguez, 2015).

De hecho, aunque el mundo afectivo de las personas «tradicionalmente, ha ofrecido una gran resistencia a ser conceptualizado» (Pérez López, 1991, p. 201), dicha conceptualización es una extensión natural de todo el sistema. Desde el modelo perezlopiano es posible conceptualizar otros tipos de mecanismos internos que facilitan las interaccionesy permiten nuevos tipos de satisfacciones que se corresponden con aquellas propias del ser humano (satisfacciones afectivas o estructurales). Estos mecanismos son tres:

10 Esto no es nuevo, ya la psicología clásica de cuño aristotélico lo conceptualizó con la distinción entre continente y virtuoso. Al respecto dice Fuentes (2007, pp. 201-202): «La persona continente es alguien que puede emitir un juicio prudencial correcto [...], que será luego ejecutado por la afectividad, siempre y cuando su razón no sea asaltada por pasiones inesperadas o vehementes en exceso. Actúa, pues, en circunstancias relativamente favorables o no intensamente adversas. Se distingue del verdadero virtuoso en que su afectividad no posee una connaturalidad respecto del auténtico bien sensible. La afectividad en el virtuoso, en cambio, está ordenada [...] desde adentro; es decir, hay un gusto y tendencia del afecto hacia el bien verdadero, o sea, siente bien del auténtico bien [...]; además, experimenta repugnancia intrínseca hacia lo que es desordenado en su objeto». En la psicología aristotélica ambas son necesarias, continencia que frena las pasiones y la virtud que ordena las pasiones hacia el fin elegido (Cfr. Garcés y Giraldo, 2014; Garcés, 2015). 
[...] para modelizar los procesos afectivos [requerimos] la introducción de tres nuevas realidades que forman parte [...] de un agente libre personal. La primera de ellas es la que llamaremos satisfacciones estructurales... [la cual] implica una necesidad de carácter lógico de que exista [lo] que llamaremos potencia afectiva. La operación de esta potencia [...] exige la introducción de un nuevo mecanismo cognoscitivo: el conocimiento experimental. (Pérez López, 1991, p. 201).

El conocimiento y la memoria percepcional activa la motivación espontánea, es decir, aquello sentido en el agente personal. El conocimiento racional, que se genera en el agente al razonar sobre los posibles resultados intrínsecos y trascendentes (Ariño, 2005, p. 9o), es activado por la libertad, operativizada por la motivación racional mediante la racionalidad y virtualidad del agente (variables de control o de autocontrol). Si el agente actúa de forma consistente y tiene éxito en su interacción (resuelve su problema), no solo habrá logrado una satisfacción percepcional, sino que tendrá un conocimiento experimental de la aplicación del plan de acción consistente (y exitoso). Ese conocimiento experimental es «el conocimiento que se tiene del estado interno de otra persona [agente reactivo] fruto de haber interaccionado con ella» (Ariño, 2005, p. 9o), el cual activará automáticamente la futura motivación espontánea (en este caso, gracias a su potencia afectiva), sintiendo no solo la necesidad de resolver su problema, sino la urgencia de considerar al otro, es decir, un tipo de satisfacción estructural. Este impulso sentido facilitará a la motivación racional futura, pero no la suplirá.
Al actuar, el agente activo sabe cómo es él mismo cuando se comporta de forma consistente. Por tanto, al interactuar con el agente reactivo induce su propia experiencia sobre el otro. Ahora ve lo que antes no veía, aquellas señales en la acción del otro que le indican algo sobre su propio estado y si este (el agente reactivo) también le toma en cuenta en sus interacciones ${ }^{11}$. Empieza a tener certidumbre experimental acerca de los motivos trascendentes que impulsarán las futuras reacciones de un determinado agente reactivo. Esa certidumbre «es algo que siente aquel agente, que lo siente respecto a un agente reactivo concreto, y que lo siente a priori de cualquier futura interacción concreta con ese agente» (Pérez López, 1991, p. 204).

Ahora bien, esa satisfacción estructural tiene diversos aspectos. Piénsese, por ejemplo, «en lo que significa añadir al disfrute de cualquier satisfacción percepcional, la seguridad de que podrá ser repetida en el futuro cuando así lo desee el agente» (Pérez López, 1991, p. 204). Este aspecto de la satisfacción estructural «orienta la acción de los agentes libres hacia el logro de estados de la organización compatibles con la maximización absoluta de las satisfacciones percepcionales» (Pérez López, 1991, p. 204). En este caso, es posible que ambos agentes tengan en cuenta el criterio de

11 «Es evidente que ese experimentar no puede tener el mismo significado que le damos al hablar de la experiencia de una interacción; el estado de la organización no puede ser objeto de una percepción directa, tal como lo es cualquier interacción» (Pérez López, 1991, p. 208). Podemos decir que el agente activo, al experimentar el éxito de su acción, tiene una motivación que le hace ver o considerar los resultados trascendentes de manera espontánea. 
consistencia, pero por motivos extrínsecos: se busca algo del otro agente (resolver cada uno sus problemas), tomando en cuenta al otro en la decisión. Si se tiene éxito, no solo se tendrá satisfacción percepcional, sino la satisfacción estructural de la seguridad de la factibilidad de las futuras interacciones.

Un segundo aspecto de la satisfacción estructural es que, además de la seguridad de la factibilidad de las futuras interacciones, tenemos una satisfacción por saberse tenido en cuenta por el otro agente, es decir, saberse amado o amor afectivo. Esto presupone que el ser humano puede actuar desinteresadamente, lo cual parece ser propio del ser humano:

Querer es desear algún bien del que carecemos o tenemos entera posesión de él. No obstante, el amar, que es también netamente humano, no parece reducirse a querer, sino que indica algo más. Efectivamente, uno no ama porque le falte algo que necesita [problema], pues amar es dar generosamente. (Sellés, 2011, p. 596).

No es solamente que el otro nos toma en cuenta en sus decisiones por resolver sus problemas, sino que nos toma en cuenta con independencia de haber resuelto él mismo sus problemas. Amor de donación o ágape le llamaban los clásicos (Polo, 1999), amor racional o efectivo le llama Pérez López. Así, este aspecto de la satisfacción estructural (amor afectivo) solo se alcanza cuando se toma el criterio de consistencia por motivos trascendentes, es decir, se toman decisiones por motivación racional por motivos trascendentes. Por un lado, al actuar el agente activo sobre el agente reactivo, tomándole en cuenta por motivos trascendentes, le ama efectivamente; por el otro, si el agente reactivo le tiene en consideración por motivos trascendentes, actuará también por amor efectivo; si ambos actúan así ambos sentirán amor afectivo. Obsérvese que no es posible acceder al amor afectivo si no se ama efectivamente. En ese sentido, si bien ambos sentirán la necesidad de actuar de tal manera que el otro resuelva sus problemas con independencia de los suyos, si lo hacen buscando el amor afectivo, en el fondo están actuando motivados por resolver un problema suyo y no del agente reactivo, con lo que estamos nuevamente ante la búsqueda de resultados extrínsecos por motivación espontánea, actuando de forma inconsistente. Este es el peligro del sentimentalismo que hay que evitar (Pérez López, 2014, p. 174). Así «el amor, en su sentido propio y pleno, es el impulso de un agente para el logro del máximo de satisfacción afectiva o afecto, es decir, para el logro de la unidad perfecta con otros agentes personales» (Pérez López, 1991, p. 271), dicho impulso puede ser afectivo (motivación espontánea) o efectivo (motivación racional).

Ahora bien, el desarrollo de la intensidad de los sentimientos es explicable por la «repetición de experimentos que confirmen el contenido de los sentimientos» (Pérez López, 2014, p. 171). Mientras más experimentos permitan verificar la hipótesis de que el otro me tiene en cuenta (teniéndole el agente activo en cuenta), se confirmará o no la hipótesis y mayor será la certidumbre del agente activo 
acerca de que la hipótesis es verdadera; por tanto, mayor será la intensidad de los sentimientos (amor afectivo) del agente activo (Pérez López, 2014, pp. 170-171). Esto es, en el fondo, un aprendizaje estructural en el agente activo: crece su autocontrol de tal forma que no solo le facilita actuar por motivación racional por motivos trascendentes, sino que le posibilita sentir cada vez más amor afectivo según tenga mayor autocontrol. Y decimos posibilita porque el amor afectivo también requiere que el agente reactivo tome decisiones por motivación racional por motivos trascendentes (depende de su grado de autocontrol). Gráficamente se puede ver en la figura 3 .

En la figura 3 puede verse que el agente A tiene un grado de autocontrol (representado por las flechas verticales continuas) más fuerte que el agente $\mathrm{B}$; por tanto, A ama efectivamente mejor que B. También se observa que puede amar afectivamente más intensamente que $\mathrm{B}$, pero no lo podrá hacer con $\mathrm{B}$ porque el amor afectivo que A siente está limitado por la capacidad de amar efectivamente de B (en otros términos, la satisfacción estructural de A está limitada por el autocontrol de B). Lo que significa que el grado de unidad, confianza mutua o amor mutuo está siempre limitado por el agente de menor autocontrol. Por otra parte, si hubiese un incremento de autocontrol por parte de B (representado por la flecha vertical punteada), el grado de amor mutuo o unidad crecería para ambos (ambos accederían a mayores cuotas de amor afectivo), por lo que conviene que $B$ logre mayor autocontrol. Además, para el agente $A$, al tener su potencia afectiva desarrollada (su motivación espontánea), le será más fácil amar efectivamente a $\mathrm{B}$, mientras que a B le costará mayor esfuerzo, hasta que pueda desarrollarla. Sin embargo, si B interactúa con un agente $C$ cuyo grado de autocontrol es menor que el suyo, podrá sentir una ayuda extra para amar efectivamente a $C$, pues tendrá su motivación espontánea desarrollada (figura 4). De esa manera B sentirá ganas de ayudar a $C$, facilitándole pensar en alternativas consistentes por motivos trascendentes y ponerlas en acción; en cambio $C$ requerirá mayor fuerza interior.

Figura 3

Grado de unidad entre dos agentes

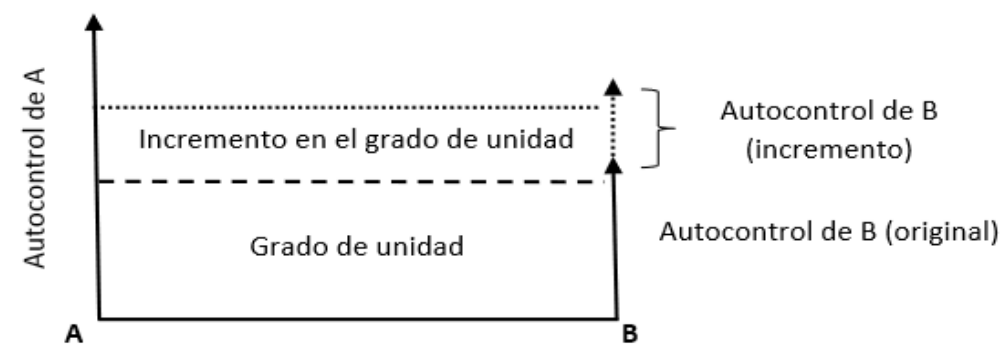

Nota: Modificado del original de Toma de decisiones y gobierno de organizaciones de Ariño, M. A., 2005, p. 102. 
Figura 4

Grado de unidad entre más de dos agentes

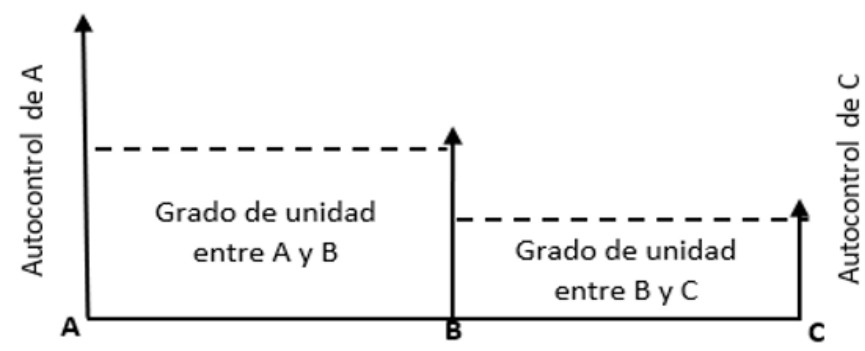

Nota: Modificado del original de Toma de decisiones y gobierno de organizaciones de Ariño, M. A., 2005, p. 103.

\section{Conclusiones}

Hemos podido exponer de manera sucinta la propuesta afectiva del modelo de la acción humana en las organizaciones de Pérez López. Con el rigor lógico que caracteriza al pensamiento de nuestro autor se ha podido observar cómo se interrelacionan las emociones con diversos rasgos que definen a una persona, a través de las nociones de motivos, motivación espontánea, motivación racional, satisfacción estructural, tipos de conocimiento, etc. A su vez, hemos podido exponer cómo se integran las emociones en la toma de decisiones y en el dinamismo afectivo en las organizaciones (en el nivel mínimo de una organización compuesta de dos personas). Se ha observado cómo, para la explicación intrínseca de la acción organizacional, se requiere de la variable afectiva, la cual facilita la relación estructural (confianza) necesaria para la interacción actual y futura de dos agentes personales. Estas nociones pueden ser complementadas a través deotras - eficacia, atractividad y unidad (Pérez López, 2014) - que no eran necesarias para esta exposición. Queda pendiente el trabajo de poder interrelacionarlas con los diferentes temas que estudia el campo del comportamiento organizacional; por ejemplo, cómo las emociones influyen en el uso del poder o la autoridad, en la comunicación, en el liderazgo, etc., lo cual será trabajo de futuras aplicaciones teóricas de este modelo hacia esos temas. Por ahora podemos decir que, si bien hay quienes lo han intentado (Ferreiroy Alcázar, 2012), aún no han realizado la conexión afectiva explícita necesaria para poder interpretar futuras investigaciones empíricas que incluyan la variable afectividad.

\section{Referencias}

Alcázar, M. (2010). Las decisiones directivas: Una aproximación antropológica al logro de eficacia y de aprendizajes positivios en las organizaciones. Tesis doctoral (promanuscrito). Pamplona, España.

Álvarez de Mon, S., Cardona, P., Chinchilla, N., Pin, J. R., et al. (2001). Paradigmas del liderazgo. Claves de la dirección de personas. Madrid: McGraw-Hill.

Argandoña, A. (2008). Integrating Ethics into Action Theory and Organizational Theory. Journal of Business Ethics, 435-446. 
Ariño, M. A. (2005). Toma de decisiones y gobierno de organizaciones. Barcelona, España: Deusto.

Ashkanasy, N. M. (2015). Emotions in organizations: A multi-level perspective. MultiLevel Issues in Organizational Behavior and Strategy, 2, 9-54.

Brennan, R. (1969). Psicología General. Madrid: Morata.

Carroll, S. (2017). El Gran Cuadro. Los orígenes de la vida, su sentido y el universo entero. Pasado y Presente.

Caruso, D. (2001). Emotionally Challenged. People Management, 19, 40-44.

Choza, J. (1988). Manual de antropología filosófica. Madrid: Rialp.

Fabro, C. (1982). Introducción al problema del hombre. La realidad del alma. Madrid: Rialp.

Ferreiro, P., y Alcázar, M. (2012). Gobierno de personas en la empresa (6.a ed.). Lima: Planeta.

Feser, E. (2006). Philosophy of mind. Oneworld Publications.

Flores-Castro, G. (2018). Ciencia para el matrimonio y la familia: Propuesta para un modelo basado en la filosofía realista. Perspectiva de familia, 3, 99-114.

Flores-Castro, G. (2018). Integración de la ética en la toma de decisiones. Un ensayo desde una perspectiva epistemológica y antropológica a partir del modelo de Pérez López. Illustro, 9, 53-80.

Fuentes, M.Á. (2007). Educar los afectos. San Rafael (Mendoza), Argentina: Ediciones del Verbo Encarnado.

Fuster, J. M. (2016). Cerebro y libertad. Los cimientos cerebrales de nuestra capacidad para elegir. Barcelona: Ariel.

Garcés, L. F. (2015). La virtud aristotélica como camino de excelencia humana y las acciones para alcanzarla. Discusiones filosóficas, 16(27), 127-146.

Garcés, L. F., y Giraldo, C. (2014). The Aristotelian continence: channeling of righteous actions in the scientist>s experiments with animals. Civilizar Ciencias Sociales y Humanas, 14(26), 199-210.

García, A. T. (2004). Una Nueva Teoría de Motivación: El Modelo Antropológico de Juan Antonio Pérez López. Revista Puertorriqueña de Psicología, 15, 123-163. García, J. Á. (2014). Antropología Filosófica. Una introducción a la Filosofía del Hombre (6.a ed.). Pamplona, España: EUNSA.

Gorrochotegui, A. (2007). Un modelo para la enseñanza de las competencias de liderazgo. edu.edu., 87-102.

Grandey, A. A. (2008). Emotions at Work: A Review and Research Agenda. En J. Barling y C. L. Cooper, The SAGE Handbook of Organizational Behavior (Vol. I: Micro Approaches, pp. 235-261). Trowbridge: SAGE.

Kangasharju, H., y Nikko, T. (2009). Emotions in Organizations. Journal of Business Communication, 46(1), 100-119. Lee, K., y Allen, J. (2002). Organizational Citizenship Behavior and Workplace Deviance: The Role of Affect and Cognition. Journal of Applied Psychology, 87(1), 131-142.

Locke, E. A. (2005). Why emotional intelligence is an invalid concept. Journal of Organizational Behavior, 26, 425-431. Locke, E. A. (2009a). Attain emotional control by understanding what emotions are. En E. A. Locke, Handbook of principles of Organizational Behavior (2nd ed., pp. 145-159). Chippenham: Wiley. 
Locke, E. A. (2009b). Itss timeto brought introspection out of the closet. Perspectives of Psychological Science, 4(1), 24-25.

Lopez Jurado, M. (2010). La decisión correcta. Barcelona: Desclée De Brouwer.

Mastenbroek, W. (200o). Organizational behavior as emotion management. En N. M. Ashkanasy, C. E. Hartel y W. Zerbe, Emotions in the Workplace: Research, Theory, and Practice (pp. 19-35). Westport: Quorum Books.

Mele, A. R. (2011). Free Will and Science. En R. Kane, Oxford Handbook of Free Will (2nd ed.). Oxford University Press. Mele, A. R. (2014). Free: Why science hasnst disproved free will. Oxford University Press.

Melé, D., y González Cantón, C. (2015). Fundamentos antropológicos de la dirección de empresas. Pamplona: Astrolabio EUNSA.

Melé, D., Chinchilla, N. M., y López-Jurado, M. (2018). The «Freely Adaptative System». Application of this Cybernetic Model to an Organization Formed by Two Dynamic Human Systems. Philosophy of Management.

Pérez López, J. A. (1991). El poder... ¿Para qué? Cuadernos de Empresa y Humanismo(29), 3-19.

Pérez López, J. A. (1991). Teoría de la acción humana en las organizaciones. La acción personal. Madrid, España: Rialp.
Pérez López, J. A. (2014). Fundamentos de Dirección de Empresas (6.a ed.). Madrid: Rialp.

Polo, L. (1999). La amistad en Aristóteles. Anuario Filosófico, 32, 477-485.

Rodríguez, F. (2015). El sujeto emocional. La función de las emociones en la vida humana. Sevilla: Thémata.

Rosanas, J. M. (2006). Methodology and Research in Management. Occasional Paper IESE Business School.

Rosanas, J. M. (2007). Beyond Economic Criteria: A Humanistic Approach to Organizational Survival. Journal of Business Ethics, 78, 447-462.

Rosanas, J. M. (2007). On the Methodology of Management Research. IESE, WP-692.

Sapolsky, R. (2017). Behave. The Biology of Humans at our Best and Worst. New York: Penguin Press.

Scheler, M. (1942). La gradación de la vida emocional. En M. Scheler, Ética (Vol. II, pp. 110-127). Madrid.

Sellés, J. F. (2011). Antropología para Inconformes (3.a ed.). España: Rialp.

Staw, B. M., y Barsade, S. G. (1993). Affect and managerial performance: A test of the sadder-but-wiser vs. happier-and smarter hypotheses. Adminnistrative Science Quaterly, 38(2), 304-331. 